\title{
Autoimmune Atrophic Chronic Gastritis
}

National Cancer Institute

\section{Source}

National Cancer Institute. Autoimmune Atrophic Chronic Gastritis. NCI Thesaurus. Code C156076.

Chronic atrophic gastritis that is caused by autoimmune destruction of parietal cells in the stomach resulting in hypochlorhydria and decreased production of intrinsic factor. 